\title{
Ácaros (Arachnida, Acari) de plantas ornamentais na região noroeste do estado de São Paulo, Brasil: inventário e descrição dos sintomas causados pelos fitófagos
}

\author{
Reinaldo J. F. Feres ${ }^{1}$, Marineide R. Vieira², Rodrigo D. Daud ${ }^{3}$, Edson G. Pereira Jr. ${ }^{2}$, \\ Gabriella F. Oliveira ${ }^{2} \&$ Cecília L. Dourado ${ }^{2}$
}

${ }^{1}$ Laboratório de Acarologia, Departamento de Zoologia e Botânica, Universidade Estadual Paulista. Rua Cristóvão Colombo, 2265, Jardim Nazareth, 15054-000 São José do Rio Preto-SP, Brasil. Pesquisador bolsista CNPq. reinaldo@ibilce.unesp.br

${ }^{2}$ Laboratório de Acarologia, Departamento de Fitossanidade, Engenharia Rural e Solos, Universidade Estadual Paulista. Caixa Postal 31, 15385-000 Ilha Solteira-SP, Brasil. marineid@ bio.feis.unesp.br

${ }^{3}$ Programa de Pós Graduação em Biologia Animal, Universidade Estadual Paulista. Bolsista Capes. rodrigodaud@yahoo.com.br

\begin{abstract}
Mites (Arachnida, Acari) on ornamental plants in northwestern São Paulo, Brazil: occurrence and injury caused by phytophagous species. Phytophagous mites living on ornamental plants growing in parks, streets and residential gardens from Ilha Solteira, State of São Paulo, were studied. Leaves of 20 plant species were sampled and some specimens of the different morphospecies found were mounted in microscopy slides with Hoyer's medium for posterior identification under phase contrast microscope. Twenty three species belonging to 15 genera of eight families were recorded. From these, 13 species are phytophagous, nine are predator and one is of unknown feeding habits. The family Tetranychidae presented the highest richness, with nine species registered, being one of these recorded for the first time after the original description and another one being registered for the first time in Brazil. Lorryia formosa Cooremann, 1958 (Tydeidae) occurred on the highest number of hosts. The host plants that harbored the highest number of mite species were Lagerstroemia indica L., Mussaenda alicia Hort. and Tabebuia sp., with six species recorded on each.
\end{abstract}

KEYWORDS. Ornamental plants; plant mites; checklist; taxonomy; urban area.

\begin{abstract}
RESUMO. Ácaros (Arachnida, Acari) de plantas ornamentais na região noroeste do estado de São Paulo, Brasil: inventário e descrição dos sintomas causados pelos fitófagos. No presente trabalho foram estudados os ácaros associados a plantas ornamentais de praças, ruas e jardins residenciais, no município de Ilha Solteira, SP. Foram amostradas folhas de 20 espécies de plantas e alguns representantes das morfoespécies de ácaros encontrados foram montados em lâminas de microscopia com o meio de Hoyer, para posterior identificação sob microscópio óptico com contraste de fases. Foram registradas 23 espécies pertencentes a 15 gêneros de oito famílias. Dessas espécies, 13 são fitófagas, nove predadoras e uma de hábito alimentar não determinado. A família com maior riqueza de ácaros foi Tetranychidae, com nove espécies, sendo uma delas registrada pela primeira vez após a descrição original e outra pela primeira vez no Brasil. Lorryia formosa Cooremann, 1958 (Tydeidae) foi a que ocorreu em maior número de hospedeiros. As plantas com as maiores riquezas de ácaros foram Lagerstroemia indica L., Mussaenda alicia Hort. e Tabebuia sp., com seis espécies registradas em cada uma.
\end{abstract}

PALAVRAS-CHAVE. Ácaros plantícolas; área urbana; inventário de espécies; plantas ornamentais; taxonomia.

A acarofauna associada a plantas ornamentais de áreas urbanas é pouco conhecida no Brasil. Nos poucos trabalhos existentes, uma ou poucas espécies de plantas foram estudadas, registrando-se grande riqueza de espécies de ácaros sobre cada uma delas. Feres (1992) descreveu Allonychus brevipenis Feres, 1992 (Tetranychidae) sobre Triplaris americana L. (pau-de-formiga, Polygonaceae), erroneamente identificada como Triplaris surinamensis Cham. (R. J. F. Feres, com. pess.), localizada na área urbana de São José do Rio Preto, SP. Já Feres et al. (2003) estudaram a diversidade e a ocorrência sazonal da acarofauna em Tabebuia roseo-alba (Ridl.) Sand. (ipê-branco, Bignoniaceae) relacionando 23 espécies associadas, enquanto que Daud et al. (2007) registraram 25 espécies em Bauhinia variegata $\mathrm{L}$. (pata-de-vaca, Leguminosae). Nos dois últimos trabalhos, também realizados no mesmo município, os autores registraram grandes infestações de ácaros fitófagos, sendo Lorryia formosa Cooreman, 1958 (Tydeidae) a espécie dominante em ambas comunidades.

Recentemente, a ocorrência de três espécies de Brevipalpus Donnadieu (Tenuipalpidae) sobre 55 espécies de plantas ornamentais foi verificada por Miranda et al. (2007), em levantamento realizado na área urbana do Distrito Federal, Brasil.

A fim de disponibilizar maiores informações sobre os ácaros de plantas ornamentais, no presente trabalho foi estudada a riqueza de ácaros em 20 espécies vegetais localizadas no perímetro urbano do município de Ilha Solteira, SP, relatando-se os sintomas causados pela infestação das espécies fitófagas registradas. 


\section{MATERIALE MÉTODOS}

$\mathrm{O}$ trabalho foi conduzido na área urbana do município de Ilha Solteira, localizado no extremo noroeste do estado de São Paulo, a $20^{\circ} 25^{\prime}$ de latitude sul e $51^{\circ} 20^{\prime}$ de longitude oeste, com altitude de $376 \mathrm{~m}$. Durante o período de dezembro de 2005 a dezembro de 2007 foram feitas amostragens mensais em 20 espécies de plantas ornamentais cultivadas em áreas verdes, jardins particulares, calçadas e canteiros da avenida central da cidade. Em cada amostragem as plantas foram vistoriadas para observação dos ácaros e dos sintomas decorrentes da infestação pelos fitófagos. As folhas que apresentaram infestações de ácaros fitófagos foram coletadas, armazenadas em sacos plásticos e levadas ao laboratório para identificação das espécies presentes. Os sintomas observados nas plantas e que puderam ser relacionados com a presença dos ácaros fitófagos, foram descritos.

No laboratório, as folhas infestadas foram colocadas em vidros contendo álcool etílico a 70\% e submetidas a agitação por cerca de 10 segundos para desprendimento dos ácaros, após o qual, foram retiradas. O conteúdo dos frascos foi examinado sob microscópio estereoscópico e representantes das diferentes morfoespécies encontradas em cada amostra foram montados em lâminas de microscopia com meio de Hoyer (Flechtmann 1975), exceto os eriofí́deos que foram montados em Hoyer's com cristais de iodo, e identificados sob microscópio com contraste de fases.

A nomenclatura adotada para as categorias superiores foi a proposta por Woolley (1988). Na apresentação dos resultados, precedendo o nome da espécie vegetal amostrada no item "Procedência do material examinado", os algarismos arábicos indicam o número de exemplares analisados e o dia em que foi realizada a coleta. Os algarismos romanos indicam o mês da coleta. O sexo e as fases de desenvolvimento são indicados através das seguintes abreviaturas: (f) fêmea e (m) macho, e (n) ninfa quando foi possível a identificação baseada nesta fase do desenvolvimento. A distribuição mundial das espécies de Tetranychidae e Phytoseiidae foi baseada em Bolland et al. (1998) e Migeon \& Dorkeld (2006), e Moraes et al. (2004), respectivamente, e conforme indicado no texto para as espécies dos demais grupos.

Os espécimes estudados foram depositados na coleção de Acari (DZSJRP) - http://www.splink.cria.org.br, do Departamento de Zoologia e Botânica, Universidade Estadual Paulista, São José do Rio Preto, SP.

\section{RESULTADOS EDISCUSSÃO}

Foram coletadas sobre 20 espécies de plantas ornamentais, 23 espécies de ácaros pertencentes a 15 gêneros de oito famílias, sendo 19 delas pertencentes a seis famílias de Actinedida (Cheyletidae, Eriophyidae, Stigmaeidae, Tenuipalpidae, Tetranychidae e Tydeidae), três espécies de Phytoseiidae (Gamasida) e somente uma de Winterschmidtiidae (Acaridida) (Tabela I).

\section{Gamasida \\ Phytoseiidae Berlese, 1916 \\ Euseius citrifolius Denmark \& Muma, 1970}

Procedência do material examinado. If (06-V), Cassia fistula $\mathrm{L}$.; $3 \mathrm{f}$ e $1 \mathrm{~m}$ (29-IV), Ixora coccinea L.; $1 \mathrm{~m}$ (31-I), 2f e $2 \mathrm{~m}$ (29-IV), $1 \mathrm{f}$ (06-V), Lagerstroemia indica L.; $1 \mathrm{~m}$ e 1n (01-IV), Lagerstroemia sp.; 1n (31-VIII), Murraya paniculata (L.) Jacq.; $1 \mathrm{~m}$ (29-IV), Pachira aquatica Aubl.; 1f (06-V), 1f (14-VII), Tabebuia sp.

Distribuição. Brasil - Bahia, Ceará, Maranhão, Mato Grosso do Sul, Minas Gerais, Paraíba, Pernambuco, Piauí, Rio Grande do Sul, São Paulo; Colômbia; Nicarágua; Paraguai e Peru.

Observações. Este ácaro ocorre sobre diversas espécies de plantas. Na região noroeste do estado de São Paulo é o predador mais freqüente e abundante (Feres \& Moraes 1998; Feres \& Nunes 2001; Daud \& Feres 2005). Embora seja um predador de outros ácaros e pequenos artrópodes, $E$. citrifolius prefere utilizar pólen vegetal como alimento (Daud \& Feres 2004; McMurtry \& Croft 1997). Gravena et al. (1994) e Furtado \& Moraes (1998) verificaram que esse ácaro pode desenvolver e reproduzir quando alimentado com Brevipalpus phoenicis Geijskes, 1939 (Tenuipalpidae), vetor da leprosedos-citros, e com Mononychellus tanajoa (Bondar, 1928) (Tetranychidae), importante praga da mandioca (Manihot esculenta Crantz), respectivamente. Devido à grande abundância e freqüência na região, e pela sua capacidade de predação de espécies-praga, E. citrifolius apresenta potencial para ser utilizado em programas de manejo integrado de pragas na região noroeste do estado de São Paulo.

\section{Euseius sibelius (DeLeon, 1962)}

Procedência do material examinado. 2f (25-I), 2f e $1 \mathrm{~m}$ (24-IV), 1f (22-VIII), Mussaenda alicia Hort.

Distribuição. Brasil - Bahia, Paraíba, Pernambuco, Piauí, Rio Grande do Sul, São Paulo; Colômbia; El Salvador; Guadalupe; Jamaica; Porto Rico e E.U.A.

\section{Euseius inouei (Ehara \& Moraes, 1998)}

Procedência do material examinado. If (31-VIII), Murraya paniculata (L.) Jacq.

Distribuição. Brasil - Rio Grande do Sul (Ferla et al. 2005); Uruguai.

Actinedida

Cheyletidae Leach, 1815

Cheletogenes sp.

Procedência do material examinado. 1n (18-X), Mussaenda alicia Hort.

Eriophyidae Nalepa, 1898

Aculus sp.

Procedência do material examinado. 74 exemplares (23-II), Tabebuia sp. 
Observações. Os ácaros foram observados de janeiro a março, com alto nível populacional em fevereiro. A infestação teve início na página inferior e, posteriormente, atingiu as duas superfícies. Folhas novas apresentaram os bordos enrolados para cima e folhas maduras exibiram intenso bronzeamento na superfície inferior.

\section{Stigmaeidae Oudemans, 1931 Zetzellia sp.}

Procedência do material examinado. 1f (06-V), Tabebuia sp.

Observações. É conhecido o hábito alimentar de Zetzellia mali (Ewing, 1917), que se alimenta de várias espécies de ácaros tetraniquídeos na América do Norte, Europa e Israel (Jeppson et al. 1975). Essa espécie é reconhecida como importante inimigo natural de ácaros-praga da macieira na América do Norte e Europa (Gerson et al. 2003).

Tenuipalpidae Berlese, 1913

\section{Brevipalpus phoenicis (Geijskes, 1939)}

Procedência do material examinado. 5f e 2 n (14-VII), Acalypha wilkesiana Müell. Arg.; 1f (06-V), Cassia fistula L; 3 f e 1n (31-I), 7f e 3n (29-IV), Lagerstroemia indica L.; 3f (25-I), 3f e 1n (24IV), 4f (22-VIII), 5f e 3n (18-X), Mussaenda alicia Hort.; 2n (03VI), Mussaenda philippica A. Rich; 1f e 1n (18-VIII), Phoenix roebelenii O'Brien; 9f e 1n (06-V), Tabebuia sp.; 4f e 2n (31VIII), Tecoma stans (L.) Juss. ex Kunth.

Distribuição. Espécie de ampla distribuição geográfica, ocorrendo sobre grande número de espécies de plantas hospedeiras. África: África do Sul; Angola; Egito; Moçambique; Malavi; Maurício; Nigéria; Quênia; Rodésia; Sudão; Tanzânia; Uganda (Meyer \& Rodrigues 1965; Rodrigues 1968; Meyer 1979); Brasil - Alagoas, Bahia, Ceará, Minas Gerais, Paraná, Pernambuco, Rio de Janeiro, São Paulo (Flechtmann 1976); Colômbia (Zuluaga \& Saldarriaga 1970); E.U.A. (Baker \& Tuttle 1987); Porto Rico (Comroy 1958).

Observações. Referido como "ácaro da leprose-doscitros", pois é o vetor da virose que causa essa patogenia nos citros (Chiavegato 1980). Registrado em seringueiras na Bahia, Mato Grosso e de São Paulo (Hernandes \& Feres 2006), e sobre plantas silvestres do noroeste do estado de São Paulo (Feres et al. 2005; Buosi et al. 2006). Essa espécie também apresenta grande capacidade de colonização de diferentes espécies de plantas freqüentemente utilizadas como cercasvivas e quebra-ventos em pomares cítricos no Brasil (Maia \& Oliveira 2004). Em Tabebuia sp. foram encontrados em grandes populações na página inferior das folhas, provocando necroses ao longo de todas as nervuras. Nesses locais pôdese observar a presença de fases ativas, quiescentes, ovos e exúvias. Sua ocorrência foi registrada de janeiro a agosto. Em $P$. roebelenii, durante o ano de 2006, essa espécie não apresentou ocorrência significativa. Entretanto, em amostragem posterior realizada em janeiro de 2008 , foi observada intensa infestação na página inferior das folhas, com grande quantidade de ácaros, ovos e exúvias. O ataque ocasionou amarelecimento generalizado do limbo foliar.

\section{Tetranychidae Donnadieu, 1875 \\ Eutetranychus banksi (McGregor, 1914)}

Procedência do material examinado. 7f, 3m e 10n (11-V), Pachira aquatica Aubl.

Distribuição. Ocorre nas Américas do Norte, Central e do Sul, Europa, Ásia e continente africano, em uma grande variedade de hospedeiros e com ampla distribuição geográfica. Já foi registrado na Argentina; Brasil; Colômbia; Costa Rica; Cuba; El Salvador; Equador; EUA; Honduras; México; Nicarágua; Panamá; Paraguai; Peru; Uruguai e Venezuela; Hawaii; Espanha e Portugal (García et al. 2003); África do Sul; Egito; Índia; Itália; Maurício; Palestina; Porto Rico.

Observações. Essa espécie apresenta variações morfológicas entre diferentes populações e hospedeiros, além de variações dentro da mesma população, em relação ao comprimento e a forma das setas dorsais (Muma et al. 1953; Pritchard \& Baker 1955; Flechtmann \& Baker 1975). Esse fato levou pesquisadores a considerarem E. banksi uma "espécie politípica" (na realidade, polimórfica). É considerada séria praga da cultura de citros nos Estados Unidos da América (Jeppson et al. 1975). No Brasil foi registrado em grande número de hospedeiros, porém sem causar danos aparentes (Bondar 1928; Flechtmann \& Abreu 1973; Feres 2000; Ferla \& Moraes 2002; Ferla et al. 2005).

Em $P$. aquatica os ácaros foram observados na página superior das folhas, no período de março a setembro, provocando amarelecimento generalizado do limbo foliar.

\section{Eutetranychus nomurai Flechtmann, 1997}

Procedência do material examinado. 26f, 10m e 2n (23-II), Phoenix roebelenii O'Brien.

Distribuição. Brasil - São Paulo (Flechtmann 1997; Bellini et al. 2005).

Observações. Em P. roebelenii os ácaros ocorreram em alta infestação nos meses de novembro e dezembro de 2006 e, posteriormente, de janeiro a abril de 2007, ocasionando intenso amarelecimento das folhas (Fig. 1). Os sintomas foram semelhantes aos observados em janeiro de 2008 em plantas altamente infestadas por $B$. phoenicis.

\section{Oligonychus poutericola Feres \& Flechtmann, 1986}

Procedência do material examinado. 6f, 8m e 1n (21-IV), Calliandra tweedii Benth.; 9f, 2m e 1n (06-V), Cassia fistula L.; 4f, 4m e 5n (21-XII), Delonix sp.

Distribuição. Brasil - São Paulo.

Observações. Essa espécie foi originalmente descrita a partir de exemplares coletados em Pouteria torta (Mart.) Radkl. Esse é o primeiro registro de $O$. poutericola após a sua descrição original.

Em C. tweedii ocorreu de abril a dezembro, com maiores populações a partir de outubro. Os ácaros desenvolveram-se, provavelmente devido à grande infestação, nas duas superfícies foliares provocando, primeiramente, uma 
descoloração generalizada, e na sequiência, bronzeamento do limbo foliar (Fig. 2). Em C. fistula ocorreu na página superior, de maio a agosto, provocando também descoloração e bronzeamento foliar. Em Delonix sp. foi registrada durante todo o ano, com maior intensidade de março a agosto, na face superior das folhas. Como conseqüência, o limbo foliar apresentou, rapidamente, um intenso bronzeamento e as plantas desfolharam nos meses de setembro e outubro. Novas brotações logo apresentaram sintomas do ataque desse ácaro.

\section{Oligonychus pratensis (Banks, 1912)}

Procedência do material examinado. 3f, $2 \mathrm{~m}$ e $7 \mathrm{n}$ (18-VIII), Phoenix roebelenii O'Brien; 7f, $5 \mathrm{~m}$ e 4n (14-XII), Washingtonia filifera (Linden) H. Wendl.

Distribuição. Argélia; China; Colômbia; Costa Rica; Egito; El Salvador; E.U.A.; Havaí; Honduras; Iraque; Madagascar; México; Paquistão; Porto Rico; Senegal e Tailândia.

Observações. Espécie cosmopolita registrada sobre grande número de hospedeiros, incluindo duas espécies de Phoenix e W. filifera. É seu primeiro registro no Brasil.

Em W. filifera foi registrada de janeiro a novembro com maiores populações nos meses mais secos do ano, de março a agosto. Os ácaros formaram densos agrupamentos na página inferior das folhas, recobertos por grande quantidade de teias. Nas áreas afetadas ocorreu um intenso amarelecimento do limbo foliar com posterior desenvolvimento de extensas necroses de coloração marrom. O papel ornamental dessas plantas ficou grandemente comprometido (Figs. 3, 4 e 5). Em $P$. roebelenii foi registrada no mês de agosto, em grande população, na página inferior das folhas que apresentaram grande quantidade de teias e de resíduos aderidos a elas, dando um aspecto de sujidade ao limbo foliar.

\section{Oligonychus aff. santoantoniensis}

Procedência do material examinado. 2f e 4n (29-IV), Ixora coccinea L. var. compacta; $8 \mathrm{f}$ e $3 \mathrm{~m}(31-\mathrm{I}), 10 \mathrm{f}, 4 \mathrm{~m}$ e $1 \mathrm{n}(25-\mathrm{III}), 4 \mathrm{f}$ e $1 \mathrm{n}$ (29-IV), 9f, 7m e 3n (06-V), Lagerstroemia indica L.; 3f, $1 \mathrm{~m}$ e $7 \mathrm{n}$ (01-IV), Lagerstroemia sp.

Observações. Em I. coccinea ocorreu na página superior das folhas, de março a novembro, provocando bronzeamento do limbo foliar. Em L. indica foi a principal espécie fitófaga, ocorrendo durante todo o ano na página superior das folhas. $\mathrm{O}$ ataque dos ácaros ocasionou intenso bronzeamento da superfície foliar que apresentou coloração preta com grande acúmulo de resíduos dando um aspecto de sujidade ao limbo (Fig. 6). As plantas infestadas desfolharam nos meses de julho e agosto, e as novas brotações, emitidas em setembro, rapidamente apresentaram esses mesmos sintomas.

\section{Tenuipalponychus tabebuiae Aguilar, Flechtmann \& Ochoa, 1991}

Procedência do material examinado. 9f, 24m e 11n (14-VII), Tabebuia sp.

Distribuição. Brasil e Costa Rica.
Observações. Os machos examinados apresentaram quatro setas no gênu I, ao invés das cinco descritas por Aguilar et al. (1991). Nas áreas de Ilha Solteira foram registrados de fevereiro a dezembro, com maiores infestações de abril a setembro. Ocorreram na página inferior das folhas, principalmente nos pontos de junção das nervuras secundárias com a central, onde há grande quantidade de tricoma. Protegidos nesse local formaram grandes agrupamentos e, em folhas intensamente atacadas, pôde-se observar muitas exúvias entre os tricomas. Como resultado da infestação ocorreu bronzeamento e queda das folhas (Figs. 7 e 8).

Em Tabebuia roseo-alba (Ridl.) Sand., a espécie ocorreu preponderantemente na estação chuvosa. As fêmeas podem entrar em diapausa, abrigando-se na face interna da base das brácteas durante a senescência das folhas (Feres \& Bellini 2002; Feres et al. 2003).

\section{Tetranychus ludeni Zacher, 1913}

Procedência do material examinado. 9f, $5 \mathrm{~m}$ e $3 \mathrm{n}$ (24-I), 1f e $1 \mathrm{n}$ (03VI), Mussaenda philippica A. Rich.; 1f (25-I), 5f e 2n (24-IV), 4f (22-VIII), 17f, $2 \mathrm{~m}$ e $2 \mathrm{n}$ (18-X), Mussaenda alicia Hort.

Distribuição. África do Sul; Alemanha; Argélia; Argentina; Austrália; Brasil; Chile; China; Colômbia; Costa Rica; Equador; El Salvador; Espanha; E.U.A.; Fiji; França;Grécia; Havaí; Honduras; Ilha Madeira; Ilhas Canárias; Ilhas Cook; Índia; Iraque; Japão; Madagascar; Malaui; Maurício; Marrocos; México; Moçambique; Nicarágua; Nova Caledônia; Nova Zelândia; Paraguai; Portugal; Peru; Polinésia Francesa; Quênia; Suíça; Taiwan; Tailândia; Tasmânia; Venezuela; Zâmbia e Zimbábue.

Observações. Em M. phillipica e M. alicia foi registrada o ano todo com vários picos populacionais, desenvolvendo-se na página inferior das folhas e provocando amarelecimento generalizado do limbo foliar.

\section{Tetranychus mexicanus (McGregor, 1950)}

Procedência do material examinado. 6f e 4m (21-II), Codiaeum variegatum (L.) A. Juss.; $12 \mathrm{f}, 3 \mathrm{~m}$ e $7 \mathrm{n}$ (31-VIII), Murraya paniculata (L.) Jacq.; $1 \mathrm{~m}$ e $1 \mathrm{n}$ (21-IV), Tecoma stans (L.) Juss. Ex Kunth.

Distribuição. Argentina; Brasil - Acre, Amazonas, Bahia, Mato Grosso, Minas Gerais, Pernambuco, São Paulo (Feres 2000; Ferla \& Moraes 2002; Buosi et al. 2006; Hernandes \& Feres 2006); Colômbia; Costa Rica, E.U.A; El Salvador; Honduras; Nicarágua; Paraguai; Peru e Uruguai.

Observações. Foi registrada de abril a outubro, formando agrupamentos na página inferior das folhas. Em M. paniculata e T. stans o ataque provocou amarelecimento do limbo foliar. Em C. variegatum as folhas, além de amareladas, ficaram encarquilhadas e com aspecto de sujidade devido ao acúmulo de detritos sobre a grande quantidade de teias.

\section{Tetranychus aff. escolaticae}

Procedência do material examinado. 14f, 3m e 1n (18-X), Musa sp.; $1 \mathrm{~m}$ (14-VI), Acalypha wilkesiana Müell. Arg. 

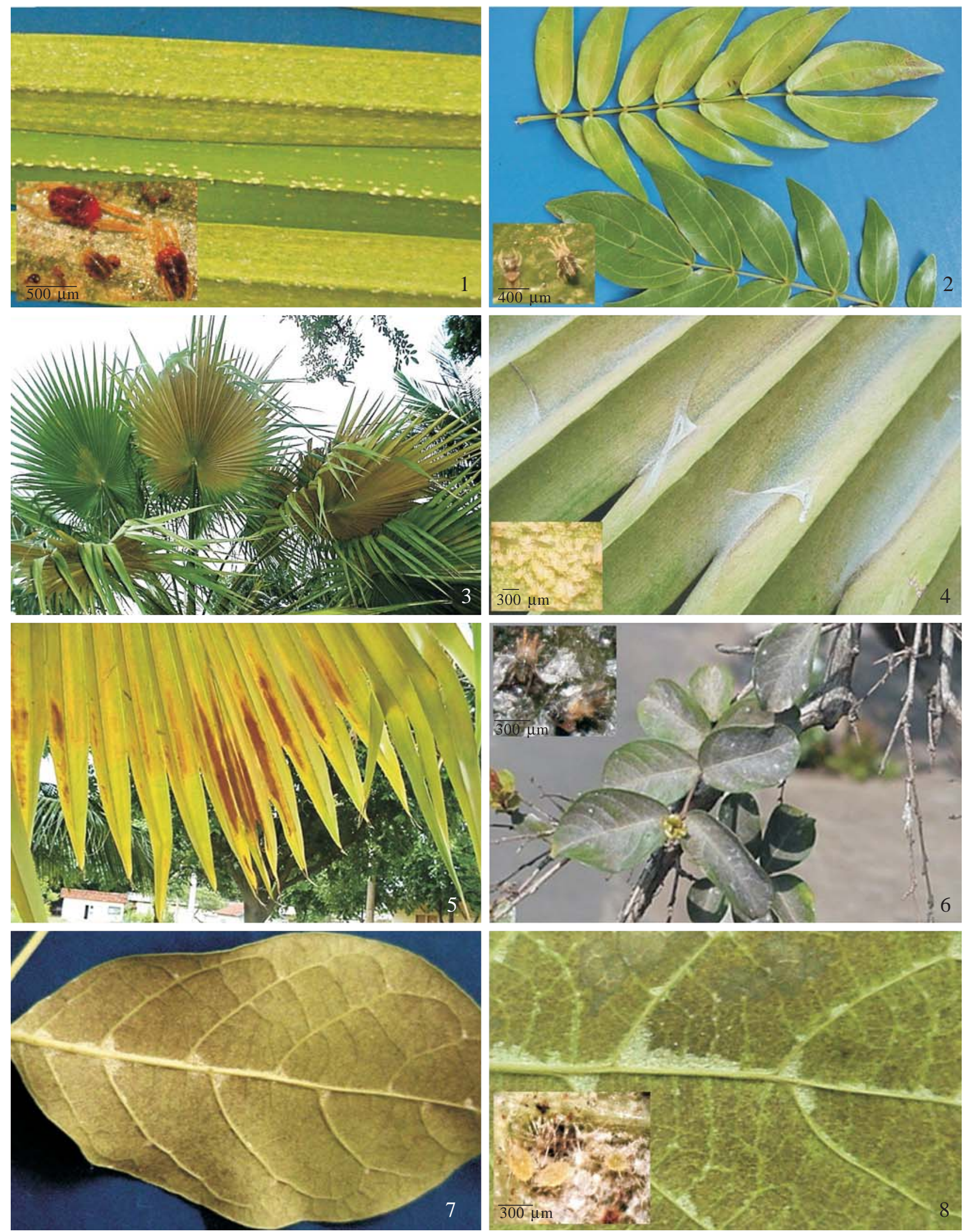

Figs. 1-8. Sintomas de infestação por ácaros fitófagos em folhas e folíolos de plantas ornamentais: 1, P. roebelenii infestada por E. nomurai (em detalhe); 2, C. tweedii infestada (acima) por O. poutericola (em detalhe) - abaixo folha normal; 3-5, W. filifera infestada por O. pratensis (em detalhe na Fig. 4); 6, L. indica infestada por $O$. aff. santoantoniensis (em detalhe); 7 e 8, Tabebuia sp. infestada por T. tabebuiae (em detalhe na Fig. 8). (Fotos: M. R. Vieira) 
Tabela I. Relação de plantas ornamentais analisadas e espécies de ácaros registradas, no município de Ilha Solteira, SP.

\begin{tabular}{|c|c|c|}
\hline \multicolumn{2}{|c|}{ Hospedeiros } & \multirow{2}{*}{ Ácaros amostrados } \\
\hline Nome científico & Nome popular & \\
\hline Arecaceae & & \\
\hline Phoenix roebelenii & tamareira anã & $\begin{array}{l}\text { Brevipalpus phoenicis } \\
\text { Eutetranychus nomurai } \\
\text { Oligonychus pratensis }\end{array}$ \\
\hline $\begin{array}{l}\text { Washingtonia filifera (Linden) H. Wendl. } \\
\text { Bignoniaceae }\end{array}$ & palmeira-de-saia-da-califórnia & Oligonychus pratensis \\
\hline Tabebuia sp. & ipê (jovem-provavelmente rosa) & $\begin{array}{l}\text { Aculus sp. } \\
\text { Euseius citrifolius } \\
\text { Zetzellia sp. } \\
\text { Brevipalpus phoenicis } \\
\text { Tenuipalponychus tabebuiae } \\
\text { Lorrvia formosa }\end{array}$ \\
\hline Tecoma stans (L.) Juss. ex Kunth & ipê-de-jardim & $\begin{array}{l}\text { Brevipalpus phoenicis } \\
\text { Tetranychus mexicanus } \\
\text { Lorryia formosa } \\
\text { Cf. Neopronematus sp. }\end{array}$ \\
\hline $\begin{array}{l}\text { Bombaceae } \\
\text { Pachira aquatica Aubl. }\end{array}$ & falso cacau & $\begin{array}{l}\text { Euseius citrifolius } \\
\text { Eutetranychus banksi } \\
\text { Lorryia formosa } \\
\text { Pronematus sp. }\end{array}$ \\
\hline $\begin{array}{l}\text { Euphorbiaceae } \\
\text { Acalypha wilkesiana Müell. Arg. }\end{array}$ & crista-de-peru & $\begin{array}{l}\text { Brevipalpus phoenicis } \\
\text { Tetranychus aff. escolaticae }\end{array}$ \\
\hline $\begin{array}{l}\text { Fabaceae } \\
\text { Cassia fistula L. }\end{array}$ & cássia-imperial & $\begin{array}{l}\text { Euseius citrifolius } \\
\text { Brevipalpus phoenicis } \\
\text { Oligonychus poutericola } \\
\text { Lorryia formosa }\end{array}$ \\
\hline $\begin{array}{l}\text { Delonix sp. } \\
\text { Leguminosae }\end{array}$ & flamboyant & Oligonychus poutericola \\
\hline $\begin{array}{l}\text { Codiaeum variegatum (L.) A. Juss. } \\
\text { Lythraceae }\end{array}$ & cróton amarelo & Tetranychus mexicanus \\
\hline Lagerstroemia indica $\mathrm{L}$ & resedá rosa & $\begin{array}{l}\text { Euseius citrifolius } \\
\text { Brevipalpus phoenicis } \\
\text { Oligonychus aff. santoantoniensis } \\
\text { Lorryia formosa } \\
\text { Lorryia sp. }\end{array}$ \\
\hline Lythraceae & & \\
\hline Lagerstroemia indica $\mathrm{L}$. & resedá rosa & $\begin{array}{l}\text { Pronematinae } \\
\text { Czenspinskia sp. }\end{array}$ \\
\hline Lagerstroemia sp. & resedá branco & $\begin{array}{l}\text { Euseius citrifolius } \\
\text { Oligonychus aff. santoantoniensis } \\
\text { Lorryia formosa } \\
\text { Czenspinskia sp. }\end{array}$ \\
\hline $\begin{array}{l}\text { Mimosaceae } \\
\text { Calliandra tweedii Benth. } \\
\text { Musaceae }\end{array}$ & esponjinha-vermelha & Oligonychus poutericola \\
\hline $\begin{array}{l}\text { Musa sp. } \\
\text { Rubiaceae }\end{array}$ & bananeira nanica & Tetranychus aff. escolaticae \\
\hline Gardenia jasminoides J. Ellis & gardênia & Lorryia formosa \\
\hline Ixora coccinea $\mathrm{L}$. & mini ixora & $\begin{array}{l}\text { Euseius citrifolius } \\
\text { Oligonychus aff. santoantoniensis } \\
\text { Lorryia formosa }\end{array}$ \\
\hline Mussaenda alicia Hort. & mussaenda-rosa & $\begin{array}{l}\text { Cheletogenes sp. } \\
\text { Euseius sibelius } \\
\text { Brevipalpus phoenicis } \\
\text { Tetranychus ludeni } \\
\text { Homeopronematus sp. } \\
\text { Lorryia sp1. }\end{array}$ \\
\hline Mussaenda frondosa $\mathrm{L}$. & mussaenda-branca & $\begin{array}{l}\text { Lorryia formosa } \\
\text { Lorryia sp1. }\end{array}$ \\
\hline Mussaenda philippica A. Rich & mussaenda-branca & $\begin{array}{l}\text { Brevipalpus phoenicis } \\
\text { Tetranychus ludeni } \\
\text { Lorryia formosa }\end{array}$ \\
\hline $\begin{array}{l}\text { Rutaceae } \\
\text { Murraya paniculata (L.) Jacq. }\end{array}$ & falsa murta & $\begin{array}{l}\text { Euseius citrifolius } \\
\text { Euseius inouei } \\
\text { Tetranychus mexicanus }\end{array}$ \\
\hline $\begin{array}{l}\text { Vitaceae } \\
\text { Petrea subserrata } \text { Cham. }\end{array}$ & flor-de-são-miguel & Lorryia formosa \\
\hline
\end{tabular}


Observações. Os caracteres diagnósticos dessa espécie correspondem aos descritos para as pertencentes ao grupo 6 de Flechtmann \& Knihinicki (2002).

Em Musa sp., foi registrada na página inferior das folhas formando densas colônias recobertas por grande quantidade de teias. Como conseqüência houve um amarelecimento generalizado do limbo foliar.

Tydeidae, 1887

\section{Homeopronematus sp.}

Procedência do material examinado. 1f e 1m (24-IV), Mussaenda alicia Hort.

Observações. Ácaros ágeis e translúcidos. Espécies desse gênero são conhecidas como predadoras de outros ácaros e de ovos de insetos (Gerson et al. 2003).

\section{Lorryia formosa Cooremann 1958}

Procedência do material examinado. 2f, $2 \mathrm{~m}$ e 4n (06-V), Cassia fistula L.; $2 \mathrm{f}, 7 \mathrm{~m}$ e $5 \mathrm{n}$ (21-IV), Gardenia jasminoides J. Ellis; $3 \mathrm{f}, 8 \mathrm{~m}$ e $3 \mathrm{n}$ (29-IV), Ixora coccinea L.; 13f, 4m e 4n (31-I), 1f, 1m e 1n (25III), 18f, $11 \mathrm{~m}, 19 \mathrm{n}$ e $1 \mathrm{~L}(29-\mathrm{IV}), 1 \mathrm{~m}(06-\mathrm{V})$, Lagerstroemia indica L.; 1f, 4m e 5n (01-IV), Lagerstroemia sp.; 7f e 7m (29-IV), Mussaenda frondosa L.; 1n (03-VI), Mussaenda philippica A. Rich; 7f, 3m e 1n (29-IV), Pachira aquatica Aubl.; 6f, 4m e 5n (21-IV), Petrea subserrata Cham.; 6f e 4n (06-V), Tabebuia sp.; 5f, 4m e 1n (21-IV), 1f e $1 \mathrm{~m}$ (31-VIII), Tecoma stans (L.) Juss. ex Kunth.

Distribuição. Argentina; Brasil; Equador; Espanha; França; Marrocos; México; Uruguai (Baker 1968); Paraguai (Aranda \& Flechtmann 1969; Flechtmann 1973).

Observações. Registrada sobre grande número de hospedeiros, sem causar dano aparente às folhas. Hernandes et al. (2006) observaram que fêmeas de $L$. formosa apresentaram desenvolvimento partenogenético telítoco, quando criadas sobre folíolos de seringueira.

\section{Lorryia sp.}

Procedência do material examinado. 1f e $1 \mathrm{~m}$ (31-I), $1 \mathrm{~m}$ (29-IV), Lagerstroemia indica L.; 1f (25-I), 1f (24-IV), Mussaenda alicia Hort.; 1m (29-IV), Mussaenda frondosa L.

\section{cf. Neopronematus sp.}

Procedência do material examinado. 1f e 1n (31-VIII), Tecoma stans (L.) Juss. ex Kunth.

\section{Pronematus sp.}

Procedência do material examinado. If e 1n (11-V), Pachira aquatica Aubl.

Observações. É conhecida a eficiência predatória de Pronematus unbiquitus (McGregor, 1923) sobre algumas espécies de eriofídeos (Perring \& McMurtry 1996). Não existem estudos sobre a potencialidade de espécies de Pronematus como inimigos naturais de ácaros fitófagos no Brasil.

\section{Pronematinae não identificado}

Procedência do material examinado. 1f (25-III), Lagerstroemia indica L.

Acaridida

Winterschmidtiidae, Oudemans 1923

Czenspinskia sp.

Procedência do material examinado. 3f e 2m (01-IV), Lagerstroemia sp.

Observações. Ácaros de movimentos lentos, de tegumento translúcido e de coloração esbranquiçada a creme. O hábito alimentar das espécies desse gênero não é conhecido, porém acredita-se que possam ser micófagas (Baker \& Wharton 1952; Krantz 1978).

Dentre as 23 espécies de ácaros associadas às plantas estudadas, foram identificadas 12 espécies nominais. Das não identificadas nominalmente, pelo menos cinco espécies podem ser novas para ciência.

A maioria das espécies amostradas é fitófaga, com 13 espécies registradas, enquanto que nove são predadoras e uma de hábito alimentar não conhecido.

A família com maior riqueza de espécies foi Tetranychidae, seguida por Tydeidae e Phytoseiidae com 9, 6 e 3 espécies de ácaros, respectivamente. Nas demais famílias apenas uma espécie foi registrada (Tabela I).

A espécie que ocorreu em maior número de hospedeiros foi $L$. formosa, encontrada sobre 11 espécies de plantas. Já o predador mais freqüente foi $E$. citrifolius, registrado sobre sete espécies vegetais (Tabela I). Ambas as espécies têm sido encontradas com freqüência em diversas plantas nativas e cultivadas no estado de São Paulo (Buosi et al. 2006; Hernandes \& Feres 2006; Daud \& Feres 2005; Feres et al. 2005; Zacarias \& Moraes 2002). Grandes infestações de $L$. formosa também têm sido registradas sobre plantas localizadas em áreas urbanas (Daud et al. 2007; Daud \& Feres 2005; Feres et al. 2003), o que é indicativo de sua grande adaptabilidade em ambientes antrópicos.

Brevipalpus phoenicis foi registrada em oito hospedeiros diferentes. Considerada praga-chave dos citros por ser vetor do vírus da leprose, sua grande capacidade de adaptação a diferentes hospedeiros é um fator de preocupação em relação ao seu controle.

Os hospedeiros que apresentaram maior riqueza de ácaros foram L. indica, M. alicia e Tabebuia sp., com seis espécies registradas em cada um (Tabela I).

Os resultados obtidos mostram a necessidade de realização de maior número de estudos taxonômicos e ecológicos sobre a comunidade de ácaros associados a essas plantas.

Agradecimentos. Ao Doutorando Fábio A. Hernandes, Programa de Pós-graduação em Biologia Animal, campus de São José do Rio Preto - UNESP, pela confecção da prancha de figuras.

\section{REFERÊNCIAS}

Aguilar, H.; C. H. W. Flechtmann \& R. Ochoa. 1991. Rediscovery and redescription of Tenuipalponychus tabebuiae (Acari: 
Tetranychidae). International Journal of Acarology 17: 113115.

Aranda C. B. R. \& C. H. W. Flechtmann. 1969. Ácaros do gênero Lorryia no Brasil e Paraguay. Anais da II Reunião da Sociedade Brasileira de Entomologia: 41-42.

Baker, E. W. 1968. The genus Lorryia. Annals of the Entomological Society of América 61: 986-1008.

Baker, E. W. \& D. M. Tuttle. 1987. The false spider mites of Mexico (Tenuipalpidae: Acari). U.S. Department of Agriculture, Technical Bulletin 1706: 1-237.

Baker, E. W. \& G. W. Wharton. 1952. An introduction to Acarology. New York, The MacMillan Company, 465 p.

Bellini, M. R.; G. J. de Moraes \& R. J. F. Feres. 2005. Ácaros (Acari) de dois sistemas de cultivo da seringueira no noroeste do Estado de São Paulo. Neotropical Entomology 34: 475-484.

Bolland, H. R.; J. Gutierrez \& C. H. W. Flechtmann. 1998. World catalogue of the spider mite family (Acari: Tetranychidae). Brill, Leiden, 392 p.

Bondar, G. 1928. Relatório. Boletim do Laboratório de Patologia Vegetal 4: 39-46.

Buosi, R.; R. J. F. Feres; A. R. Oliveira; A. C. Lofego \& F. A. Hernandes. 2006. Ácaros plantícolas (Acari) da "Estação Ecológica Paulo de Faria”, Estado de São Paulo, Brasil. Biota Neotropica 6: 1-20.

Chiavegato, L. G. 1980. Ácaros da cultura dos citros, p. 469-501. In: Rodriguez, O. \& F.C.P. Viégas (Coord.) Citricultura brasileira. Campinas, Fundação Cargill, 739 p.

Comroy, H. L. 1958. A preliminary survey of the plant mites of Puerto Rico. Journal of Agriculture of the University of Puerto Rico 42: 39-144.

Daud, R. D. \& R. J. F. Feres. 2004. O valor de Mabea fistulifera Mart. (Euphorbiaceae), planta nativa do Brasil, como reservatório para o predador Euseius citrifolius Denmark \& Muma (Acari, Phytoseiidae). Revista Brasileira de Zoologia 21: 453-458.

Daud, R. D. \& R. J. F. Feres. 2005. Diversidade e flutuação populacional de ácaros (Acari) em Mabea fistulifera Mart. (Euphorbiaceae) de dois fragmentos de mata estacional semidecídua em São José do Rio Preto, SP. Neotropical Entomology 34: 191-201.

Daud, R. D.; R. J. F. Feres \& R. Buosi. 2007. Ácaros (Arachnida: Acari) associados a Bauhinia variegata L. (Leguminosae) no noroeste do estado de São Paulo. Neotropical Entomology 36: 322-325.

Feres, R. J. F. 1992. Allonychus brevipenis, a new species from Triplaris surinamensis Cham. in Brazil with new host record for $A$. braziliensis (McGregor) and A. reisi Paschoal (Acari: Tetranychidae). International Journal of Acarology 18: 299301.

Feres, R. J. F. 2000. Levantamento e observações naturalísticas da acarofauna (Acari: Arachnida) de seringueiras cultivadas (Hevea spp., Euphorbiaceae) no Brasil. Revista Brasileira de Zoologia 17: $157-173$.

Feres, R. J. F. \& G. J. de Moraes. 1998. Phytoseiid mites (Acari: Phytoseiidae) from woody areas in the state of São Paulo, Brazil. Systematic and Applied Acarology 3: 125-132.

Feres, R. J. F. \& M. A. Nunes. 2001. Ácaros (Acari, Arachnida) associados a euforbiáceas nativas em áreas de cultivo de seringueiras (Hevea brasiliensis Muell. Arg., Euphorbiaceae) na região noroeste do estado de São Paulo, Brasil. Revista Brasileira de Zoologia 18: $1253-1264$.

Feres, R. J. F. \& M. R. Bellini. 2002. Ocorrência de diapausa em Tenuipalponychus tabebuiae Aguilar, Flechtmann \& Ochoa 1991 (Acari, Tetranychidae) no Estado de São Paulo, Brasil. Revista Brasileira de Zoologia 19: 219-224.

Feres, R. J. F.; A. C. Lofego \& A. R. Oliveira. 2005. Ácaros plantícolas (Acari) da "Estação Ecológica do Noroeste Paulista", estado de São Paulo, Brasil. Biota Neotropica 5: 1- 4 .

Feres, R. J. F.; M. R. Bellini \& D. de C. Rossa-Feres. 2003. Ocorrência e diversidade de ácaros (Acari, Arachnida) associados a Tabebuia roseo-alba Sand. (Bignoniaceae), no município de São José do Rio Preto, São Paulo, Brasil. Revista Brasileira de Zoologia 20: 373-378.

Ferla, N. J. \& G. J. de Moraes. 2002. Ácaros (Arachnida, Acari) da seringueira (Hevea brasiliensis Muell. Arg.) no Estado do Mato Grosso, Brasil. Revista Brasileira de Zoologia 19: 867-888.

Ferla, N. J.; M. M. Marchetti \& J. C. Siebert. 2005. Acarofauna (Acari) de erva-mate (Ilex paraguariensis St. Hil.: Aqüifoliaceae) no estado do Rio Grande do Sul. Biociências 13: 133-142.

Flechtmann, C. H. W. 1973. Lorryia formosa Cooremann, 1958 - Um ácaro dos citros pouco conhecido no Brasil. Ciência e Cultura 25: $1179-1181$.

Flechtmann, C. H. W. 1975. Elementos de Acarologia. São Paulo, Livraria Nobel S. A., 344 p.

Flechtmann, C. H. W. 1976. Preliminary report on the false spider mites (Acari: Tenuipalpidae) from Brazil and Paraguay. Proceedings of Entomological Society of Washington 78 : 58-64.

Flechtmann, C. H. W. 1997. Mite (Arthropoda: Acari) associates of palms (Arecaceae) in Brazil. III. Eutetranychus nomurai n.sp. (Tetranychidae) from Attalea phalerata Mart. International Journal of Acarology 23: 269-273.

Flechtmann, C. H. W. \& D. K. Knihinicki. 2002. New species and new record of Tetranychus Dufour from Australia, with a key to the major groups in this genus based on females (Acari: Prostigmata:Tetranychidae). Australian Journal of Entomology 41: $118-127$.

Flechtmann, C. H. W. \& E. W. Baker. 1975. A report on the Tetranychidae (Acari) of Brazil. Revista Brasileira de Entomologia 19: 111-122.

Flechtmann, C. H. W. \& J. M. Abreu. 1973. Ácaros fitófagos do Estado da Bahia, Brasil. Ciência e Cultura 25: 244-251.

Furtado, I. P. \& G. J. de Moraes. 1998. Biology of Euseius citrifolius, a candidate for the biological control of Mononychellus tanajoa (Acari: Phytoseiidae, Tetranychidae). Systematic and Applied Acarology 3: 43-48.

García, E.; A. L. Márquez; S. Orta \& P. Alvarado. 2003. Caracterización de la presencia de Eutetranychus banksi (MacGregor) y Eutetranychus orientalis (Klein) en el Sur de España. Phytoma 153: 90-95.

Gerson, U.; R. L. Smiley \& R. Ochoa. 2003. Mites (Acari) for pest control. Oxford, Blackwell Science Ltd, 537 p.

Gravena, S.; I. Benetoli; P. H. R. Moreira \& P. T. Yamamoto. 1994. Euseius citrifolius Denmark \& Muma predation on citrus leprosis mite Brevipalpus phoenicis (Geijskes) (Acari: Phytoseiidae: Tenuipalpidae). Anais da Sociedade Entomológica do Brasil 23: 209-218.

Hernandes, F. A. \& R. J. F. Feres. 2006. Review about mites (Acari) of rubber trees (Hevea spp., Euphorbiaceae) in Brazil. Biota Neotropica 6: 1-24.

Hernandes, F. A.; R. J. F. Feres \& F. Nomura. 2006. Biological cycle of Lorryia formosa (Acari, Tydeidae) on rubber trees leaves: a case of thelytoky. Experimental and Applied Acarology 38: 237-242.

Jeppson, L. R.; H. H. Keifer \& E. W. Baker. 1975. Mites injurions to economic plants. Los Angeles, University California Press, 614p.

Krantz, G. W. 1978. A manual of acarology. Corvallis, Oregon State University Book Stories, Inc. p. i-viii, 1-508 p.

Maia, O. M. A. \& C. A. L. Oliveira. 2004. Capacidade de colonização de Brevipalpus phoenicis (Geijskes) (Acari: Tenuipalpidae) em cercas-vivas, quebra-ventos e plantas invasoras. Neotropical Entomology 33: 625-629.

McMurtry, J. A. \& B. A. Croft. 1997. Life-styles of phytoseiid mites and their roles in biological control. Annual Reviews Entomology 42: 291-321.

Meyer, M. K. P. S. 1979. The Tenuipalpidae (Acari) of Africa with keys to the world fauna. Entomology Memoir. Department of Agricultural Technical Services 50, 135 p.

Meyer, M. K. P. S. \& M. da C. Rodrigues. 1965. Acari associated with cotton in Southern Africa (with reference to other plants). Garcia de Orta 13: 195-226.

Migeon, A. \& F. Dorkeld. 2006. Spider Mites Web: a comprehensive database for the Tetranychidae. < http://www 1.montpellier. inra.fr/CBGP/spmweb >. Acesso: 06/08/2009.

Miranda L. C.; D. Návia \& J. C. V. Rodrigues. 2007. Brevipalpus mites 
Donnadieu (Prostigmata: Tenuipalpidae) associated with ornamental plants in Distrito Federal, Brazil. Neotropical Entomology 36: 587-592.

Moraes, G. J. de; J. A. McMurtry; H. A. Denmark \& C. B. Campos. 2004. A revised catalog of the mite family Phytoseiidae. Zootaxa 434: 1-494.

Muma, M. H.; H. Holtzberg \& R. M. Pratt. 1953. Eutetranychus banksi (McGregor) recently found on citrus in Florida (Acarina: Tetranychidae). Florida Entomologist 4: 141-144.

Perring, T. M. \& J. A. McMurtry. 1996. Other Predatory Arthropods, p. 471-479. In: Lindquist, E. E., M. W. Sabelis \& J. Bruin (Eds.). Eriophyiod mites: Their biology, natural enemies and control. Amsterdan, Elsevier Science, 790 p.

Pritchard, A. E. \& E. W. Baker. 1955. A revision of the spider mites family Tetranychidae. Memoirs series, Vol. 2, San Francisco, Pacific Coast Entomological Society, 472 p.

Rodrigues, M. da C. 1968. Acarina de Moçambique - Catálogo das espécies relacionadas com a agricultura. Agronomica Moçambicana 2: 215-256.

Woolley, T. A. 1988. Acarology: Mites and Human Wellfare. New York, Wiley, 484 p.

Zacarias, M. S. \& G. J. de Moraes. 2002. Mite diversity (Arthropoda: Acari) on Euphorbiaceous plants in three localities in the State of São Paulo. Biota Neotropica 2: 1-12.

Zuluaga, C. \& V. Saldarriaga. 1970. Reconocimiento, identificacion y algunas observaciones sobre dinamica de poblaciones de acaros, en citricos del valle del Cauca. Acta Agronomica 20: 114-141. 